\title{
The Disaggregation Algorithm in Nonintrusive Load Monitoring
}

\author{
Chuleui Hong \\ Department of Human Intelligence Information Science, Sangmyung University, \\ Seoul, 110-743, Korea \\ hongch@smu.ac.kr
}

\begin{abstract}
The nonintrusive load monitoring on individual household appliances by using energy disaggregation algorithm is a technique to infer the energy consumption of each appliance by analyzing the changes in power supplied to the household. The technology presented in this study provides consumers with energy-saving methods such as standby power cut-off, device abnormality, and purchase of power-saving products by informing consumers of the energy use and time zones for each electric appliances. In this paper, the real and reactive power quantities are measured from the total amount of power used, and compare them with the previously saved power signature of each household electric device to identify the devices that are being used. In this experiment, the disaggregation accuracy showed good performance at $95.3 \%$ on five appliances and seven electric devices.
\end{abstract}

Keywords: Appliances Identification, Disaggregation, NILM, Real and Reactive Power

\section{Introduction}

The nonintrusive load monitoring (NILM) is a technology that estimates energy consumption of household appliances currently used by analyzing the changes in power supplied from the incoming line of house [1][2]. Meters are equipped in the incoming line to investigate the different use of home appliances in consumer homes. This method saves money in terms of cost rather than measuring current consumption by installing measuring instruments on each household appliance

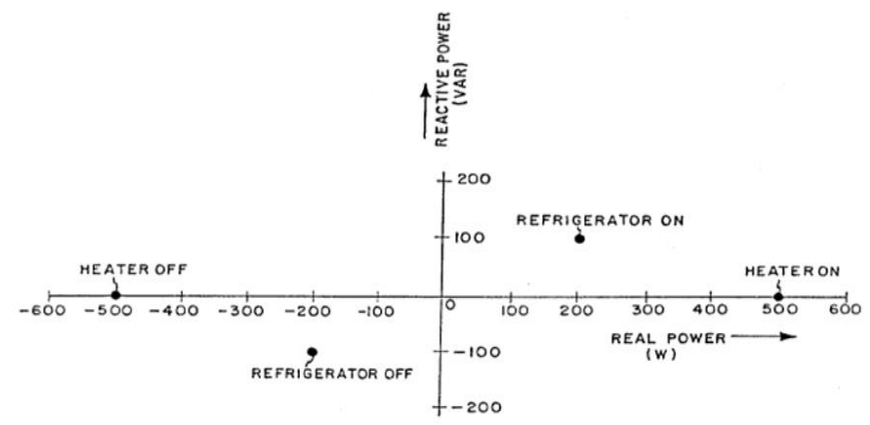

Figure 1. The reactive power changes of different electric devices

Article history:

Received (March 6, 2019), Review Result (July 9, 2019), Accepted (November 12, 2019) 
The nonintrusive individual electrical appliance load monitoring system can measure the real and reactive powers. Thus, as shown in Figure 1, although the reactive power of electric motor changes significantly when it is switched on and off, the reactive power of the heater makes little difference, so the data on reactive power provides another way to determine the use of household devices.

A nonintrusive individual home appliance load monitoring system can detect the power use of different types of household appliances by analyzing continuous changes in a total real power, as shown in Figure 2. The size of change in real power is defined as an edge. These edges are modeled like finite-state machines to determine which devices are being used through the energy disaggregation algorithm [3].

The energy disaggregation algorithm determines which devices are being used by discomposing the total power into the individual power usage of each household appliance as a statistical approach. Each device is identified because it has its own characteristics in power.

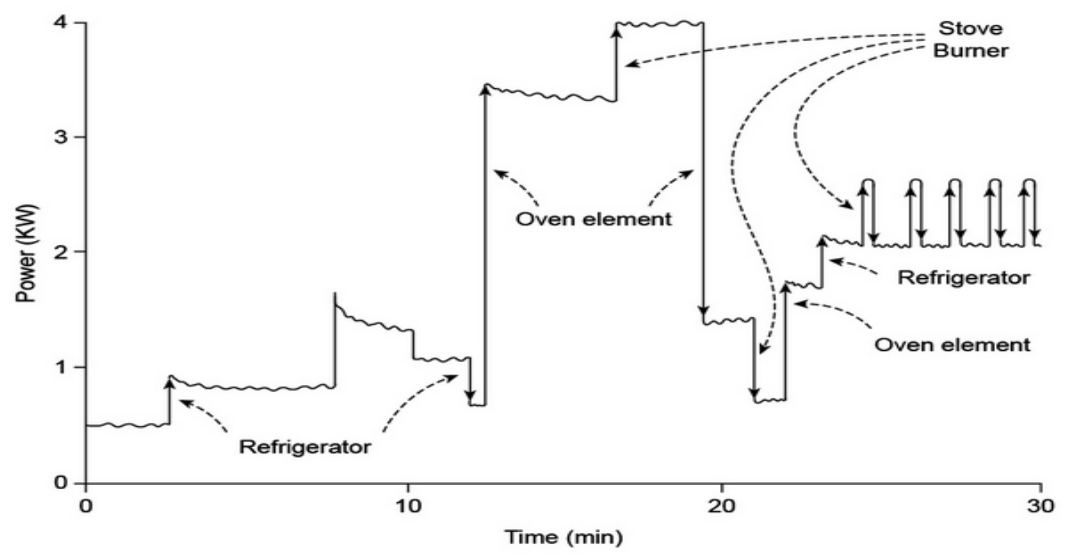

Figure. 2. The disaggregation of electric devices

\section{The disaggregation algorithm in NILM}

The disaggregation algorithm uses a signature comparison technique for the electrical power of every household appliances which are built from physical measurements on appliances in advance. When arbitrary signals are detected, this algorithm finds the most similar pattern in a pool of existing signature information.

This statistical approach utilizes electrical data characteristics, such as power and cycles. In terms of power, both real and reactive powers are useful. Along with the real power, reactive power improves to identify appliances with similar real powers in a low sampling cycle of less than $60 \mathrm{~Hz}$ [4][5][6]. This is because appliances have different reactive power independent of the real power.

\subsection{Frequency of data collection}

Depending on the frequency of data collection, two distinct characteristics can be found. First, the high frequency can provide the ability to detect even the home appliances that are always turned on, but it is difficult in the low frequency. This is be-cause many devices have their own waveforms, and sampling cycles greater than $60 \mathrm{~Hz}$ can see actual alternating current and voltage waveforms, and can take ad-vantage of the harmonic characteristics. Second, it takes more time to obtain sufficient data for classification in the low frequency below $60 \mathrm{~Hz}$. 
Thus, for example, data collected by the low frequency method may require data ranging from one week to several months for the classification of devices used.

\subsection{Performance of disaggregation algorithm}

Let $P(t)$ be total power consumption during a specific time interval, $t$. Then we identify the type of appliances and their operating conditions by disassembling specific power signals $\left(p_{i}\right)$ of each device from the total power consumption $P(t)$. Although we can easily collect data in low frequency, we need more sophisticated disaggregation algorithm. In this study, 20 samples were performed per second in the low cycle range.

$$
P(t)=p_{1}(t)+p_{2}(t)+p_{3}+\cdots \cdot+p_{n}(t)
$$

The optimization approach stores data sets or load signatures classified in the device characteristics database, and then it minimizes error by searching the nearest the amount of power $P(t)$ from all possible combinations of power demand for home devices. Let $v_{n}$ be total power of the combination of devices and $\mathrm{v}$ be the measured total power, then we can infer the currently used devices, class $\left(v_{n}\right)$, from the nearest $v_{n}$.

$$
\operatorname{Class}\left(v_{n}\right)=\min \mid{ }^{4} v_{n}-v \|
$$

\subsection{Classification in a real/reactive of power plane}

This algorithm uses a frequency histogram to perform classifications on the real $(P)$ and reactive power $(Q)$ plane and to generate classifiers. The combined classifier is used to break down the total power load into device-level power load estimates, and then we can classify the loads and identify the types of devices and their operational states. After the voltage is normalized for a given time zone, a 2D histogram is completed with 1 watt unit on a P-Q plane.

The process of the algorithm is as follows.

1) After the clusters for each state of the loads are organized, the clusters are classified clearly by the histogram-thinning process.

2) Classify each cell in histogram and calculate the Probability Mass Function (PMF) for the cells. Each cell represents the state of device.

3) Repeat the above steps for each device and calculate the maximum likelihood classifier for each state.

4) Calculate all possible combinations of each device state and calculate the joint probability for each combination using the probability mass function of each class to create a single classifier for the composite load.

5) The combined classifier is used to break down the total power load to the device-specific power load estimate to identify the type of devices and their operational status.

\subsection{Classification using edge state}

The measured power data for each device is studied based on the naive Bayesian method to find the most possible state of each device. Analyze the measured power usage patterns and separate the states of the device. From the power data of each state we can measure the edge that represents the power changes in each pair of states of each device. The probability that the device is in a particular state can be estimated when the previously known edge is detected.

Then a Bayes' theory is applied to create a classifier. Train classifiers for each of the other devices and use trained classifiers to break down devices from overall power usage. It is assumed that the state of each device is completely independent. 
In summary, the classification algorithm is as follows.

1) The status set $S$ can be made up of single or multiple devices. So set $S$ indicates the status of each device $D_{i}$ is in a particular state $s_{i}$. This means that the disaggregated state for each device is already known.

2) Calculate the probability of each state set $S$ from the training data to find the most possible solution.

3) $\operatorname{Pr}(E=e \mid S)$ : Assuming that only one device may change states at a time with the probability that a corresponding edge will occur during a state change, The probability can be calculated that a state $\mathrm{S}$ is changed from any state by the edge $e$. Calculate the frequency of the edge, $e$, of all the edges, E, that makes the state change to $S$

4) Signature generation of known devices: Based on the size of changing edges for each device, create a signature database, train classifiers, and disaggregate devices from measured total power loads.

The signature preparation phase measures the total amount of power for the sole and multiple devices. For the unmeasured power, this algorithm estimates the amount of power using interpolated methods. The measured powers pass through the medium filter to eliminate low and high frequency noise and calculate the probability density function (PDF) for every power uses for each device to produce a histogram in 1 watt bin.

\section{The experimental results}

In this experiment, five different types of appliances and seven different devices were used as shown in Table 1. Data collection was performed 20 times per second using Yokokawa's WT210. The experiment developed a signature by obtaining the change in the edge state of the real power and the real/reactive power plane of each sample during the learning phase. The sample is made up of one or more composite devices.

\subsection{Creation of signature}

We measure the real and reactive powers data for each sample in the steady-state state.

1) Real and reactive power measurement

2) Make a frequency histogram on the real power $(P)$ and reactive power $(Q)$ plane to generate classifiers.

3) To calculate the probability that the device is in a particular state when there is a detected edge for real power, a classifier is created using the Bayes' theory formula.

4) Train classifiers for each sample and use trained classifiers to disaggregate devices from overall power usage.

\subsection{Disaggregation}

We remove the noise by using the medium filter from the measurement data and obtain the steady-state. In case of unmeasured intervals of power, an estimated value is generated using the interpolated method from the measured information. Since the sum of the real power of each device can be used as the sum of the real power of the corresponding composite devices, calculate the sum of the real power when using the composite device from the measured real power value. Using the combined real/reactive power and edge classifiers, the total measured power is disaggregated down into estimates of the power of each device, while identifying the type of device and its operational states. 

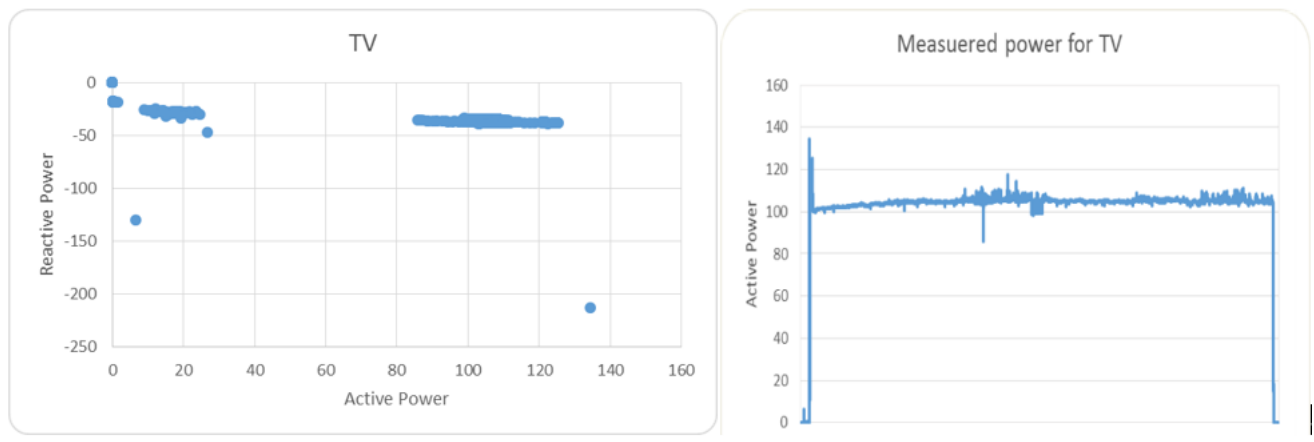

Figure 4. The P-Q plane and edge signature of each device

The figure 4 shows signatures for each home appliance. The results of experiments on seven devices are shown in Table 1 . The average accuracy was calculated at $95.25 \%$. In the case of audio, the accuracy was most incorrectly recognized at $80 \%$, which, unlike other appliances, the states of the edge were not correctly recognized, resulted in the false recognition when multiple set-top boxes are used simultaneously. Therefore, more research will be expected on the edge changes of audio to create the more precise signatures.

In addition, there were occasional wrong disaggregation about heater recognition errors as using lights together, but they were soon correctly recognized. This seems necessary to research further on the $P-Q$ power plane than this experiment.

Table 1. Accuracy of devices

\begin{tabular}{|c|c|c|c|}
\hline No. & Type & Device & Accuracy \\
\hline 0 & Set-top & BKO-100 & 98.5 \\
\hline 1 & & SMT-C5010 & 97.2 \\
\hline 2 & TV & 32LK451 & 99.5 \\
\hline 3 & & UN32EH4000F & 99.7 \\
\hline 4 & Audio & MM-E330D & 80.0 \\
\hline 5 & Light & OSRAM 20EX-L & 98.2 \\
\hline 6 & Heater & HEFW-2150 & 93.6 \\
\hline
\end{tabular}

\section{Conclusions}

In this paper, total amount of power used were collected non-intrusively from the incoming line of the house. The disaggregation algorithm to identify the household devices in use was developed by extracting the real and reactive power variations from the collected power changes. The presented power disaggregation can find out devices currently used from signature characteristics that are unique to each house-hold device, such as distribution on the real/reactive power plane of each household device and the amount of variation in the real power generated by changes in the state of the device. In particular, the disaggregation accuracy was increased to $95.3 \%$ using the class of $P-Q$ plane $t$ and he Bayesian technique on the state of the edges indicating the real power change.

It can be said that the algorithms presented in this study proved superior in terms of the recognition of low-power appliances such as set-top boxes and audio, considering the accuracy of most existing papers at around $90 \%$ [7]. 
Future research will improve disaggregation accuracy by taking into account of temporary on/off state changes and high order harmonic values as well as real and reactive power. We will further research on the deep learning on this area, which is currently being studied [8].

\section{Acknowledgements}

This research was supported by a 2018 Research Grant from Sangmyung University.

\section{References}

[1] S. Giri, and M. Berges, "An energy estimation framework for event-based methods in Non-Intrusive load monitoring," Energy Conversion and Management, vol.90, pp.488-498, (2015) DOI: 10.1016/j.enconman.2014.11.047

[2] S. Chae, and J. Park, "A study of Non-Intrusive appliance load identification algorithm using complex sensor data processing algorithm," The Journal of The Institute of Internet, Broadcasting and Communication, April, vol.17, no.2, pp.199-204 (2017) DOI: 10.7236/JIIBC.2017.17.2.199

[3] I. Abubakar, S. N. Khalid, M. W. Mustafa, H. Shareef, and M. Mustapha, "Application of load monitoring in appliances energy management - A review," Renewable and Sustainable Energy Reviews, vol.67, pp.235-245 (2017) DOI: 10.1016/j.rser.2016.09.064

[4] R. Bonfigli, E. Principi, M. Fagiani, M. Severini, and F.Piazza, "Non-intrusive load monitoring by using active and reactive power in additive Factorial Hidden Markov Models", Applied Energy, vol.208, pp.1590-1607 (2017) DOI: 10.1016/j.apenergy.2017.08.203

[5] S. Son, "Hybrid approach for home electricity consumption monitoring," International Conference on Future Information \& Communication Engineering, vol.7, no.1, pp.279-282 (2015)

[6] C. Hong, "A distributed hybrid algorithm for glass cutting”, Journal of DCS, February, vol.19, no.2, pp.343349 (2018)

[7] S. Hosseini, K. Agbossou, S. Kelouwani, and A. Cardenas, "Non-intrusive load monitoring through home energy management systems: A comprehensive review," Renewable and Sustainable Energy Reviews, vol.79, pp.1266-1274 (2017) DOI: 10.1016/j.rser.2017.05.096

[8] M. Figueiredo, A. Almeida, and B. Ribeiro, "Home electrical signal disaggregation for non-intrusive load monitoring (NILM) systems,” Neurocomputing, vol.96, pp.66-73 (2012) DOI: 10.1016/j.neucom.2011.10.037 